\title{
Efeitos de doses de raios-gama na obtenção de mutantes de coloração de inflorescência de crisântemo (Dendranthema grandiflorum)
}

\author{
ANGELA MARIA BOERSEN (1); AUGUSTO TULMANN NETO (2); RODRIGO ROCHA LATADO (2); \\ PEDRO CÉSAR DOS SANTOS (3)
}

\begin{abstract}
RESUMO
A indução de mutações com a finalidade de melhoramento genético tem sido bastante utilizada em plantas ornamentais em vários países, incluindo o Brasil, o que resultou no lançamento de novas cultivares. Em experimentos de mutagênese induzida, muitos são os fatores que podem afetar a frequiência e os diferentes tipos de mutantes obtidos, bem como o tipo de quimerismo observado (quimeras setoriais, mericlinais ou periclinais). Um dos principais fatores, certamente, é a dose de mutagênico empregada. Assim, recomenda-se que sejam realizados experimentos prévios de avaliação da sensitividade das espécies ao mutagênico, antes de tratar um grande número de propágulos. No presente trabalho, o objetivo foi avaliar os efeitos de diferentes doses de raios-gama, na freqüência de obtenção de mutantes de coloração de inflorescência e no tipo de quimera resultante, após a irradiação de estacas não-enraizadas de crisântemo cv Cherry Dark. As doses utilizadas foram de 0, 10; 12,5; 15; 17,5; 20; 25 e 30 Gy de raios-gama, em sete experimentos consecutivos. Em todos os experimentos avaliou-se a sobrevivência, número total de mutantes, o número de mutantes de coloração de inflorescência e o tamanho dos setores mutados, enquanto que a altura de plantas só foi avaliada no primeiro. Observou-se uma tendência a decréscimo linear na altura de plantas e quadrático, na taxa de sobrevivência, em função do aumento das doses de raios-gama. O GR50 (redução de 50\% no crescimento) para essa variedade foi estimado em 18,1 Gy. As maiores freqüências de obtenção de plantas mutantes, incluindo as de coloração de inflorescência, foram observados nas doses de 10 e 12,5 Gy, com tendência à redução nas doses maiores. Mutantes periclinais, com todas as inflorescências da planta mutada para coloração, somente foram observados a partir de 15 Gy e tiveram uma tendência a aumento na sua freqüência de aparecimento com o aumento da dose.
\end{abstract}

Palavras-chave: mutação, irradiação, ornamental, melhoramento genético

\section{ABSTRACT}

Dose effect gamma-irradiation in obtaining color mutants of inflorescence of chrysanthemum (Dendranthema grandiflorum). Mutation induction in ornamental plant breeding is intensively used in several countries including Brazil, resulting in the release of several new cultivars. Mutation induction can affect the frequency and spectrum of the resulting mutants as well as the types of chimerism (sectorial or mericlinal or periclinal chimeras). Among these, the dose used in the mutagenic treatment is of high importance. Therefore, preliminary experiments evaluating sensitivity of the material to be used are indispensable. The objective of this study was to evaluate the effect of different doses of gamma-rays on mutation frequency in color of inflorescence and type of chimerism of non-rooted cuttings of chrysanthemum 'Cherry Dark'. The doses tested were 0,$10 ; 12.5 ; 15 ; 17.5 ; 20 ; 25$ and 30 Gy of gamma-rays in seven consecutive experiments. Survival number, total number of mutants, number of mutants of inflorescence color and type of chimerism were evaluated. Plant height was evaluated only in the first experiment. Tendency of linear decrease in plant height and quadratic tendency in survival percentage was observed with increased doses of the mutagen. The GR50 (reduction of 50\% in the growth) for this variety was estimated to be $18.1 \mathrm{~Gy}$. Higher frequencies of mutants, including inflorescence color, were observed in the doses of 10 and 12.5 Gy, with a tendency of reduction in higher doses. Periclinal mutants were observed in 15 Gy and upper doses. The observed tendency was of increasing frequency of mutations with increasing dose.

Key words: mutation, irradiation, ornamental, plant breeding

\section{INTRODUÇÃO}

A indução de mutações com a finalidade de melhoramento genético tem sido utilizada em várias espécies vegetais, incluindo as plantas ornamentais. Seu impacto pode ser medido não apenas pelas 2.252 novas cultivares de diferentes espécies, liberadas oficialmente para cultivo até o ano de 2000, em dezenas de países (MALUSZYNSKI et al., 2000), mas também pelo grande impacto econômico resultante destes cultivos (AHLOOWALIA et al., 2004).
Entre os mutagênicos, os raios-gama foram os mais empregados em trabalhos de mutagênese induzida (64\% dos casos) e as plantas ornamentais ou decorativas foram as que obtiveram o maior número de cultivares mutantes liberadas, com 25\% do total (MALUSZYNSKI et al., 2000). Esse sucesso deve-se ao fato de haver uma maior facilidade na seleção de mutações de coloração e de morfologia e, também pelo fato de as plantas ornamentais geralmente terem ciclo curto e uma alta heterozigosidade, o que possibilita que mutações recessivas (as mais freqüentes) possam ser reconhecidas imediatamente

\footnotetext{
(1) Parte da Dissertação de Mestrado da primeira autora.

(2) Centro de Energia Nuclear na Agricultura/USP, CP 96, 13400-970, Piracicaba(SP). Autor correspondente, e-mail: tulmann@cena.usp.br.

(3) Centro APTA Citros "Sylvio Moreira” - IAC, CP.4, 13490-970, Cordeirópolis (SP).

(4) UNESP - Campus de Ilha Solteira, Av. Brasil, 56, 15385-000, Ilha Solteira(SP).
} 
(BROERTJES \& VAN HARTEN, 1988a).

O sucesso dessa técnica em ornamentais é particularmente notável na cultura de crisântemo, na qual foram liberadas 210 novas cultivares até o ano de 2004, e muitas dessas permaneceram por vários anos no mercado, contribuindo com milhões de dólares para os produtores (AHLOOWALIA et al., 2004). Tais mutantes destacaramse não apenas pelas colorações obtidas, diferentes da variedade original usada para o tratamento, como também pelos novos formatos de inflorescência, precocidade, resistência ao frio, entre outras características.

No Brasil, apesar de ser usada apenas recentemente em ornamentais, a indução de mutações com uso de raiosgama já produziu novas cultivares de crisântemo de corte, que foram liberadas aos produtores e consumidores (TULMANN NETO \& LATADO, 1997; ADAMES et al., 1999). Devido à crescente importância das ornamentais no Brasil (FLORA BRASILIS, 2002), o melhoramento de plantas por indução de mutações, pode trazer boas perspectivas ao setor (TULMANN NETO \& LATADO, 1999).

Em experimentos de mutagênese induzida, vários são os fatores que podem afetar a freqüência e os tipos de mutantes obtidos, bem como o tipo de quimerismo que pode ocorrer nos mutantes (quimeras setoriais, mericlinais ou periclinais). Entre esses fatores, podem ser citados o tipo e a dose do mutagênico, o material vegetativo a ser tratado, o método de condução das plantas pós-tratamento, o tipo da mutação selecionada, o método de seleção da mutação, a estabilidade da variedade usada, e outros (BOROJEVIC, 1970; MICKE, 1970; GOTTSCHALK, 1970). Como destacam BOERTJES \& VAN HARTEN (1988a), uma vez que ocorra mutação com o uso do mutagênico, o quimerismo automaticamente ocorre se mais de uma célula está presente no momento do tratamento. Esses autores citam ainda que quimeras do tipo mericlinal, por exemplo, não são estáveis e que, do ponto de vista do melhoramento, outros métodos devem ser usados para se obter mutantes não quiméricos (sólidos) ou quimeras periclinais.

A sensitividade aos mutagênicos varia entre e dentro de uma mesma espécie. Assim, recomenda-se que sejam realizados experimentos prévios de avaliação da sensitividade ao mutagênico, antes de se tratar um grande número de propágulos (GAUL, 1970). O objetivo destes geralmente é definir mais precisamente uma dose apropriada para os experimentos de mutagênese induzida. Esses experimentos têm a vantagem de serem breves, contudo, avaliam os efeitos fisiológicos do mutagênico nos propágulos, ao invés dos efeitos genéticos (mutações).

No presente trabalho, o objetivo foi avaliar os efeitos de diferentes doses de raios-gama, na freqüência de obtenção de mutantes de coloração de inflorescência e no tipo de quimera resultante, após a irradiação de estacas não-enraizadas.

\section{MATERIAL E MÉTODOS}

As estacas não-enraizadas de crisântemo de vaso cv Cherry Dark foram cedidas pela Empresa Dekker de Wit Ltda (Mogi-Guaçu, SP). Essa variedade geralmente é cultivada em vasos e tem como características principais: grande número de inflorescências por planta, inflorescências cor de rosa, plantas com altura entre 10 a $15 \mathrm{~cm}$ e precocidade de florescimento (média de sete semanas).

Os experimentos foram conduzidos parcialmente nos laboratórios do CENA/USP, em Piracicaba (SP) e nos campos experimentais da Empresa Dekker de Wit Ltda.

Foram realizados sete experimentos consecutivos, com a irradiação de estacas não-enraizadas de crisântemo, de tamanho médio de $4,5 \mathrm{~cm}$, com quatro ou cinco folhas, utilizando-se do irradiador Gamma-cell 220 do CENA/ USP, com taxa de dose média variando entre 129,2 e 144,8 Gy/h.

No primeiro experimento foram aplicadas as doses de 0, 10; 15; 20; 25 e 30 Gy (taxa de dose de 144,8 Gy/h) de raios-gama, num total de 60 estacas não-enraizadas para cada tratamento. Aos 30 dias após a irradiação, seguindose a indicação de LATADO (1993), realizou-se a medição da altura das plantas. A avaliação dos efeitos das doses de radiação na altura de plantas foi feita por meio de análise de regressão polinomial.

Nos seis experimentos seguintes foram aplicadas as doses de 0,$10 ; 12,5 ; 15 ; 17,5 ; 20 ; 25$ e 30 Gy de raiosgama. O número de estacas irradiadas não foi o mesmo em todos os experimentos, sendo o número mínimo utilizado em cada experimento de 60 estacas para o controle e 140 estacas, para cada dose de radiação. Em todos os experimentos o delineamento utilizado foi o de blocos ao acaso com cinco repetições. Na época de florescimento, em todos os experimentos foram anotadas a sobrevivência das plantas.

Os dados de altura de plantas e taxa de sobrevivência foram submetidos à análise de variância e de regressão polinomial, utilizando-se da versão 9.1 do programa SAS, 1985. Para tanto foram usados os modelos de regressões seqüenciais com inclusão dos efeitos linear, quadrático e cúbico com intuito de utilizar o significativo de maior coeficiente de determinação.

Os procedimentos descritos a seguir foram comuns aos sete experimentos.

Para acelerar o enraizamento, as estacas foram parcialmente imersas em solução aquosa contendo 0,25\% de Ácido Indolbutírico (AIB), antes do plantio nos vasos. Em cada vaso foram colocadas três ou quatro estacas, em substrato comercial.

Os vasos foram transferidos para estufas, para o enraizamento, crescimento e florescimento das plantas. Aproximadamente, 35 dias após a irradiação, foi realizada a remoção do de ápice caulinar das plantas, com o objetivo de promover o desenvolvimento dos ramos laterais. Para o desenvolvimento vegetativo utilizou-se fotoperíodo longo (16 horas de luz/8 horas de escuro) e para a indução de florescimento fotoperíodo curto ( 8 horas luz/16 horas de escuro), como descrito com mais detalhes por BOERSEN (2003).

As avaliações do total de mutantes morfológicos e de coloração de inflorescência foram realizadas sempre durante o florescimento, examinando-se individualmente cada inflorescência das plantas e selecionando as com alguma alteração. Foram considerados como mutantes 
morfológicos os que tinham inflorescências com formato diferente, em comparação com o controle, e os mutantes de coloração foram considerados os que demonstravam nítidas diferenças na coloração da inflorescência em comparação com o controle (cor rosa). Foram consideradas- como plantas mutantes totais as com mutações na morfologia da inflorescência e/ou mutações na coloração da inflorescência.

As plantas mutantes classificaram-se de acordo com o tipo de quimera obtido, em: a) Quimera periclinal plantas mutantes com todas as inflorescências contendo coloração única e diferente da original; b) Quimera mericlinal - plantas mutantes cujas inflorescências apresentavam a cor original e uma ou mais colorações mutantes.

As plantas com coloração idêntica à planta original foram consideradas como não mutadas.

Os dados obtidos com as avaliações dos mutantes morfológicos e de coloração de inflorescência nos sete experimentos foram agrupados.

\section{RESULTADOS E DISCUSSÃO}

A análise de variância de altura de planta de crisântemo no primeiro experimento revelou significância para o fator doses de radiação $(F=59,09 \mathrm{p}<0,0001)$. As análises de regressões seqüenciais com modelo polinomial, incluindo os efeitos lineares, quadráticos e cúbicos, indicaram significância apenas para o modelo linear. Sendo assim, fez-se uma regressão com apenas esse efeito, obtendo-se os parâmetros $a=5,594(\mathrm{t}=22,49, \mathrm{p}<0,0001)$ e $b=-0,154(\mathrm{t}=-11,97, \mathrm{p}<0,0003)$. Constatou-se decréscimo na altura de planta devido ao aumento nas doses de raios-gama (Figura 1), cujos dados se ajustaram à equação $\mathrm{y}=-0,154 \mathrm{x}+5,594\left(\mathrm{R}^{2}=0,97\right)$.

$\mathrm{O}$ efeito da redução da altura de plantas, em conseqüência do aumento da dose de raios-gama, já era esperado, pois se trata- de um efeito fisiológico bastante observado quando estacas ou mudas são tratadas com mutagênicos. Desse modo, tem sido um parâmetro utilizado para seleção de doses em trabalhos com indução de mutação (TULMANN NETO \& LATADO, 1997).

De acordo com os dados obtidos e utilizando a equação citada acima, pode-se estimar a dose de 18,15 Gy, como sendo capaz de reduzir em 50\% a altura de plantas (GR50) dessa variedade. O GR50 estimado se encontra próximo ao calculado por TULMANN NETO \& LATADO (1997), em pesquisa envolvendo indução de mutações em crisântemo de corte cv Repin rosa, que foi 20 Gy. BROERTJES \& VAN HARTEN (1988b), apresentaram lista de cultivares de crisântemo obtidas por indução de mutação in vivo e afirmaram que as doses utilizadas nesses experimentos variaram entre 10 e $25 \mathrm{~Gy}$.

Na tabela 1 são relatados os dados de sobrevivência de plantas, total de plantas mutantes e plantas mutantes contendo mutações de coloração de inflorescência, obtidas nos sete experimentos (dados agrupados).

Assim como observado para a altura de plantas, a análise de variância foi significativa também para sobrevivência de plantas. Entretanto, nesse caso, a equação de regressão que melhor se ajustou foi a que incluiu efeitos lineares e quadráticos, cujos parâmetros foram $a=97,926$ $(\mathrm{t}=11,09, \mathrm{p}<0,0001), b_{1}=0,9527(\mathrm{t}=0,83, \mathrm{p}<0,4450)$ e $b_{2}=-0,1321(\mathrm{t}=-3,70, \mathrm{p}<0,0140)$. Observa-se uma tendência à redução da sobrevivência de plantas de forma quadrática, devido ao aumento da dose do mutagênico (figura 2), chegando à letalidade total na dose de 30 Gy.

A literatura constata uma grande variação no que se refere à dose letal para tratamento in vivo de crisântemo com raios-gama. BOWEN (1965) citou que a dose letal deveria se situar em 70 Gy de raios-gama, para a irradiação de gemas axilares, enquanto que BROERTJES (1966) considerou que a dose letal deveria ser superior a $80 \mathrm{~Gy}$. Já LATADO (1993) obteve alta taxa de sobrevivência utilizando dose de 32,5 Gy de raios-gama em estacas enraizadas com proteção das raízes à ação da radiação, mas com avaliação 30 dias após o tratamento.

Como na presente pesquisa não houve a proteção das raízes e, além disso, realizou-se a avaliação da sobrevivência apenas durante o florescimento das plantas. Então, pode- se supor que todas as doses de mutagênico, incluindo a de $30 \mathrm{~Gy}$, tenham afetado mais intensamente as estacas e por um maior tempo, ocasionando maior letalidade.

Observou-se que, em valores absolutos, as maiores freqüências de obtenção de plantas mutantes e de plantas com mutações de coloração de inflorescência corresponderam às doses de 10 e 12,5 Gy, chegando ao máximo de $79,1 \%$ de plantas mutantes, no total de plantas avaliadas, e 68,7\% de plantas mutantes contendo diferentes colorações de inflorescência, na dose de 12,5 Gy. Nas doses superiores observou-se uma tendência à queda gradual, em ambos os parâmetros, até a dose de 30 Gy, em que não houve plantas sobreviventes (tabela 1).

Nas doses superiores, a redução da freqüência total de mutantes pode ser explicada pelo aumento da letalidade observada nessas doses. Já as reduções nas freqüências de mutantes de coloração de inflorescência observadas nessas doses, poderiam ser creditadas ao modo de ação da radiação gama. Segundo ICHIKAWA et al. (1970), doses baixas de raios-gama devem ser utilizadas em crisântemo para induzir preferencialmente mutantes de coloração de inflorescência, pois ocasionam maior freqüência de mutações pontuais nas células. Enquanto que doses maiores devem ser utilizadas para se obter mutantes morfológicos (de formato e tamanho de inflorescência, por exemplo), que geralmente têm células contendo aberrrações cromossômicas e/ou perda de cromossomos inteiros.

As explicações sobre os mecanismos envolvidos na mutagênese para coloração em crisântemo, as doses ideais a serem utilizadas e as conseqüentes freqüências e tipos de mutantes obtidos, há tempos vêm sendo discutidas (BOWEN, 1964) e ainda não foram suficientemente esclarecidas (BROETJES \& VAN HARTEN, 1988). O que existe de concordância nesses trabalhos é que as variações morfológicas e/ou colorações obtidas a partir da mutagênese de uma cultivar podem resultar de vários fatores, tais como: mutações gênicas, aberrações cromossômicas ou rearranjamento de camadas celulares em cultivares quiméricas. Nesse último caso, o mutagênico poderia expor um quimerismo já existente na variedade 
original, permitindo a observação de novas colorações.

Pelas tabelas apresentadas verifica-se que um grande número de mutantes foi observado nos vários tratamentos, enquanto que nas 420 plantas do controle, nenhum mutante, morfológico ou de coloração, foi observado. Esse fato já é uma indicação de que as variações observadas não foram devidas ao ambiente, pois do contrário também deveriam aparecer nas plantas do controle. Além disso, como descrito por BOERSEN (1993), por meio de estaquia de várias plantas, em que foram observados tais mutantes, verificou-se que a característica selecionada foi mantida na planta obtida. Além disso, os mutantes selecionados têm características morfológicas ( por exemplo, número de pétalas dobradas, pétalas arredondadas etc..) ou de coloração bem distintas da cultivar original (branca, vermelha, salmão etc..). Somase a isso o fato de que em trabalhos anteriores dos autores, com indução de mutação em crisântemo, mutantes semelhantes aos do presente trabalho foram obtidos (ADAMES et al. 1999; LATADO, 1993; TULMANN NETO e LATADO. 1997).

Uma das razões para o grande número de mutantes de coloração observado em todas as doses utilizadas nesse trabalho deve-se à coloração rosa da cultivar utilizada. Tal coloração é citada como uma das melhores na indução de mutação em crisântemo (BROERTJES, 1966) e tem propiciado elevadas freqüências de mutantes de coloração em vários trabalhos encontrados na literatura, inclusive em alguns já realizados no Brasil com essa cultura (TULMANN NETO \& LATADO, 1997). No entanto, como relatado anteriormente, não se pode descartar a hipótese de que muitas dessas novas colorações observadas e classificadas como provenientes de mutações, podem ter sido originadas a partir de reagrupamentos ou rearranjos das camadas histogênicas anteriormente existentes na planta, que foram afetadas (parcialmente ou totalmente eliminadas) pelo tratamento com o mutagênico (NYBOM, 1961; BROERTJES \& VAN HARTEN, 1988c).

Analisando-se o efeito das diferentes doses no tamanho de setores mutados (Tabela 2), verificou-se que os mutantes periclinais, isto é, apresentáramos com todas as inflorescências da planta com a mesma coloração mutada, somente foram observados a partir da dose de 15 Gy e tiveram uma tendência a aumento na sua freqüência de aparecimento, em razão do aumento da dose. Esses tipos de quimeras são de interesse em plantas de propagação vegetativa devido à sua maior estabilidade, embora somente mutantes não quiméricos sejam realmente estáveis (DONINI, 1984).

Os dados obtidos neste trabalho estão de acordo com o observado por BOERTJES \& VAN HARTEN (1988). Esses autores citam que, em doses baixas, esperase uma alta sobrevivência de plantas e o aparecimento de setores mutados menores (setores mericlinais, por exemplo), e em doses altas, a sobrevivência diminui, mas houve o aumento na porcentagem de mutantes periclinais.

Quanto aos diferentes tipos de mutantes de coloração, nos sete experimentos foi possível a observação de diversas colorações originadas das várias doses de raiosgama, tais como: amarela, bege, branca, laranja, vermelha, salmão, bronze e etc..., e com várias tonalidades para cada cor, exemplos: amarelo-alaranjada, amarelo-limão, amareloouro e outras. Na tabela 3 estão citados os números de diferentes colorações que apareceram nas diversas doses para os mutantes quiméricos periclinais, nos quais todas as inflorescências da planta têm coloração diferente da cultivar original. Houve uma variação de duas (25 Gy) a nove cores (17,5 Gy e $20 \mathrm{~Gy}$ ) o que indica que, entre as novas cores, nem todas foram observadas nas diversas doses.

É interessante destacar que, entre as diversas cores observadas tanto nos mutantes quiméricos periclinais com nos mericlinais (esses últimos não relatados no presente trabalho), embora a maioria já seja encontrada na família de variedade Cherry, surgiram mutantes de cor vermelha, ainda não observados nessa cultivar.

Sete mutantes, para morfologia ou coloração de inflorescência, com potencial para comercialização foram selecionados para participar de ensaios de avaliação de desenvolvimento agronômico (BOERSEN, 2003). Entre os que foram selecionados pelo aspecto da coloração, um com cor salmão (originado do tratamento com $10 \mathrm{~Gy}$ ), dois com cor avermelhada (um obtido com a dose de 15 e o outro com 20 Gy) e dois com cor vermelho-alaranjada (um obtido com a dose de 17,5 Gy e o outro com 20 Gy).

Com base nos resultados verificou-se que, nas condições desse trabalho, sem levar-se em conta o tipo de quimera resultante, houve uma faixa de doses de raiosgama em que à medida em que se aumenta a dose aumentase a porcentagem de mutantes de coloração observada, ocorrendo posteriormente uma diminuição nas doses maiores (tabela 1). Já, para os mutantes periclinais, embora não tenham ocorrido nas doses menores, houve um aumento gradativo e contínuo na sua porcentagem, em função do aumento da dose (tabela 2). Isso poderia levar à recomendação de doses altas para a obtenção desses tipos de mutantes (periclinais), os quais, como descrito anteriormente, são de grande interesse devido à sua estabilidade.

No entanto, sabe-se que existe um maior risco de ocorrência de mutações múltiplas nas plantas, quando submetidas a doses elevadas de mutagênico (HANSEL 1968). Especialmente para o caso de plantas de propagação vegetativa, mutações múltiplas devem ser evitadas devido ao processo de propagação vegetativa que perpetua tanto as mutações desejáveis como as indesejáveis (DONINI \& MICKE, 1984). Então, no caso do aparecimento de mutantes contendo características desejáveis, mas com a presença de outras mutações, geralmente ocorre uma perda de interesse no seu uso para fins comerciais.

Levando-se em conta os valores de GR50, como é utilizado por alguns autores que trabalham com indução de mutações, e os aspectos citados anteriormente, se o objetivo for a obtenção de mutantes periclinais de crisântemo, com a modificação da coloração em todas as inflorescências da planta, pode-se recomendar o uso da dose de 18,15 Gy ou doses ao redor da GR50, tais como: 17,5 e 20 Gy. Tais doses propiciaram uma elevada freqüência e uma ampla variação nos tipos diferentes de colorações de inflorescência, bem como uma alta freqüência de mutantes periclinais, com todas as inflorescências das plantas mutadas. Como citado 
anteriomente, nesse intervalo de doses foram selecionados três dos cinco mutantes de coloração mais promissores, incluídos nos ensaios de avaliação. O que reforça tal recomendação.

\section{CONCLUSÕES}

Nas estacas não-enraizadas de crisântemo da cultivar Cherry Dark irradiadas com 10; 12,5; 15; 17,5; 20; 25; e 30 Gy de raios-gama, a dose de 30,0 Gy foi letal e existe uma tendência à redução linear de altura de planta e, quadrática, da taxa de sobrevivência de plantas, em função do aumento da dose de mutagênico.

A dose que causa uma redução de $50 \%$ na altura das plantas (GR50) provenientes de estacas não-enraizadas de crisântemo foi definida como 18,15 Gy.

Observa-se uma tendência à redução no número de plantas mutantes quiméricas mericlinais observadas e um aumento no número de mutantes periclinais, em função do aumento da dose de mutagênico.

As doses entre 15 e 20,0 Gy induziram o maior número de diferentes tipos de mutantes de coloração de inflorescência periclinais.

\section{REFERÊNCIAS}

ADAMES, A.H.; LATADO, R.; CAMARGO, .M.; TULMANN NETO, A. Posição da gema axilar e a indução de mutação em mudas de crisântemo (Dendranthema grandiflora Tzvelev). Scientia Agrícola, v.56, n.4, p.939945, 1999.

AHLOOWALIA, B.S.; MALUSZYNSKI, M. NICHTERLEIN, K. Global impact of mutation-derived varieties. Euphytica, n.135, p.187-204, 2004.

BOERSEN, A.M. Indução de mutação por raios-gama a partir de mudas de crisântemo (Dendranthema grandiflorum) de vaso visando-se a obtenção de novas cultivares. 2003. 152p. Dissertação (Mestrado) - Escola Superior de Agricultura “Luiz de Queiroz”, Universidade de São Paulo, Piracicaba.

BOROJEVIC, K. Factors influencing the mutant spectrum and the quality of mutants: Differences depending on the genotype. In: Manual on Mutation Breeding, International Atomic Energy Agency, Vienna-Austria, 1970, p. 125-126.

BOWEN, H.J.M. Mutations in horticultural Chrysanthemums. Radiation Botany, v.5, p.695-700, 1965. Supplement.

BROERTJES, C. Mutation breeding of Chrysanthemum. Euphytica, v.15, p.156-162, 1966

BROERTJES, C.; VAN HARTEN, A. General introduction: The use of mutation for crop improvement. In: Applied mutation breeding for vegetatively propagated . Amsterdam: Elsevier Scientific Publishing, 1988a. p.3-13.

BROERTJES, C.; VAN HARTEN, A. Ornamental Crops: Chrysanthemum. In: Applied mutation breeding for vegetatively propagated. Amsterdam: Elsevier Scientific Publishing, 1988b. p.162-175.

BROERTJES, C.; VAN HARTEN, A. Shoot apices: Organization and post-irradiation behavior. In: Applied mutation breeding for vegetatively propagated crops. Amsterdam: Elsevier Scientific Publishing, 1988c. p.2943.

DONINI, B.; MICKE, A. Use of mutations in improvement of vegetatively propagated crops. In: REGIONAL SEMINAR ON THE UTILIZATION OF INDUCED MUTATIONS FOR CROPS IMPROVEMENT FOR COUNTRIES IN LATIN AMERICA, 1,1984, Lima, Induced mutations for crop improvement in Latin America. Vienna: IAEA, 1984. p.19-98.

FLORA BRASILIS. Relatório do diagnóstico da produção de flores e plantas ornamentais brasileiro. Campinas: IBRAFLOR; BRAZIL; APEX, 2002. CDRom.

GAUL, H. Mutagen effects observable in the first generation: Plant injury and lethality. In: Manual on Mutation Breeding, International Atomic Energy Agency, Vienna-Austria, 1970, p.85-90.

GOTTSCHALK, W. Factors influencing the mutant spectrum and the quality of mutants: Pleiotropy and linkage. In: Manual on Mutation Breeding, International Atomic Energy Agency, Vienna-Austria, 1970, p.128-129.

HANSEL, H. An estimate of the rate of chlorophyll mutations in barley taking account of multiply mutated $M_{1}$ nuclei. In: Mutations in plant breeding II, International Atomic Energy Agency, Vienna-Austria, 1968, pp 139-151.

ICHIKAWA, S.; YAMAKAWA, K. SEKIGUCHI, F.; TATSUNO, T. Variation in Somatic chromosome number found in radiation-induced mutants in Chrysanthemum morifolium cv. Yellow Delaware and Delaware. Radiation Botany, 1970, v.10, p.557-562.

LATADO, R.R. Indução e uso de mutações in vivo e in vitro no melhoramento do Chrysanthemum morifolium Ram. 1993. 103p. Dissertação (Mestrado em Agronomia) Escola Superior de Agricultura "Luiz de Queiroz", Universidade de São Paulo, Piracicaba.

MALUSZYNSKY, M.; NICHTERLEIN, K.; VAN ZANTEN, L.; AHLOOWALIA, S. Officially released mutant varieties - The FAO/IAEA data base. Mutation Breeding Review, 2000, v.12, p.1-84.

MICKE, A. Factors influencing the mutant spectrum and the quality of mutants: type of mutagen and dose. In: Manual on Mutation Breeding, International Atomic Energy Agency, Vienna-Austria, 1970, p.126-128.

NYBOM, N. The use of induced mutation for the improvement of vegetatively propagated crops. In: Mutation and Plant Breeding. National Academy of Sciences, Publication 891, Washington, D.C., 1961, p. 252-294.

SAS. SAS/STAT User's guide v. 1 SAS Institute Inc. Cary, 
North Carolina, 1995.

TULMANN NETO, A.; LATADO, R.R. Indução de mutação in vivo no melhoramento de crisântemo (Dendranthema grandiflora Tzvelev) cv. Repin Rosa. Pesquisa Agropecuária Brasileira, v.32, n.11, p.1153-1158, 1997.
TULMANN NETO, A.; LATADO, R.R. Indução de mutações para o melhoramento de ornamentais e as perspectivas de sua utilização no Brasil. Revista Brasileira de Horticultura Ornamental, v.5, n.1, p.1-11, 1999.

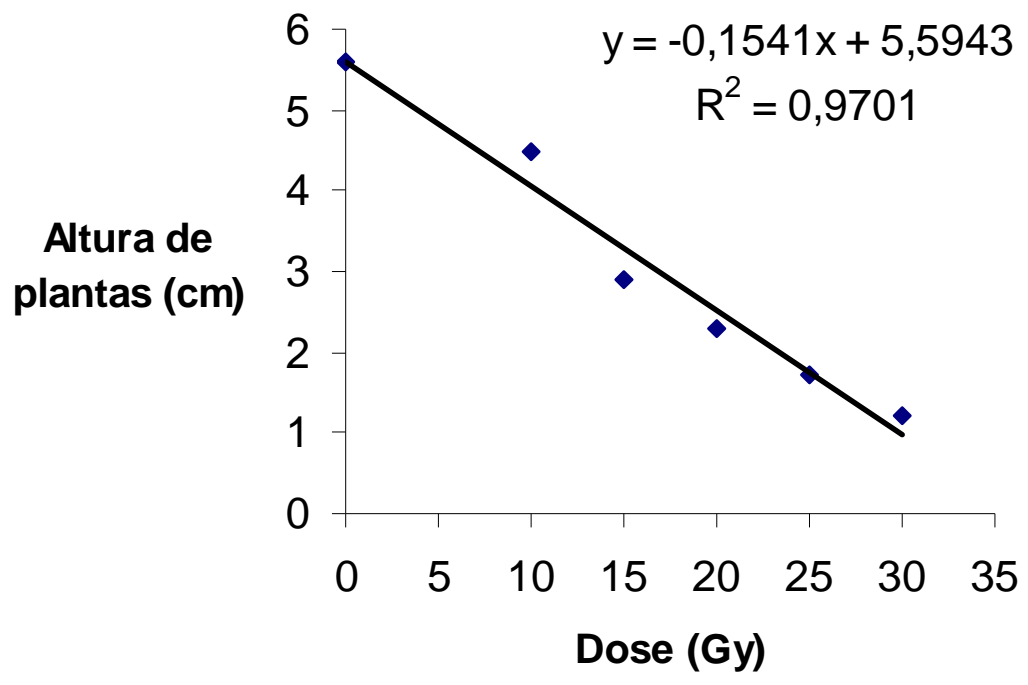

Figura 1. Altura das plantas da cultivar de crisântemo Cherry Dark, provenientes da irradiação de estacas não-enraizadas, com várias doses de raios-gama. (Avaliação realizada 30 dias após a irradiação, no experimento 1).

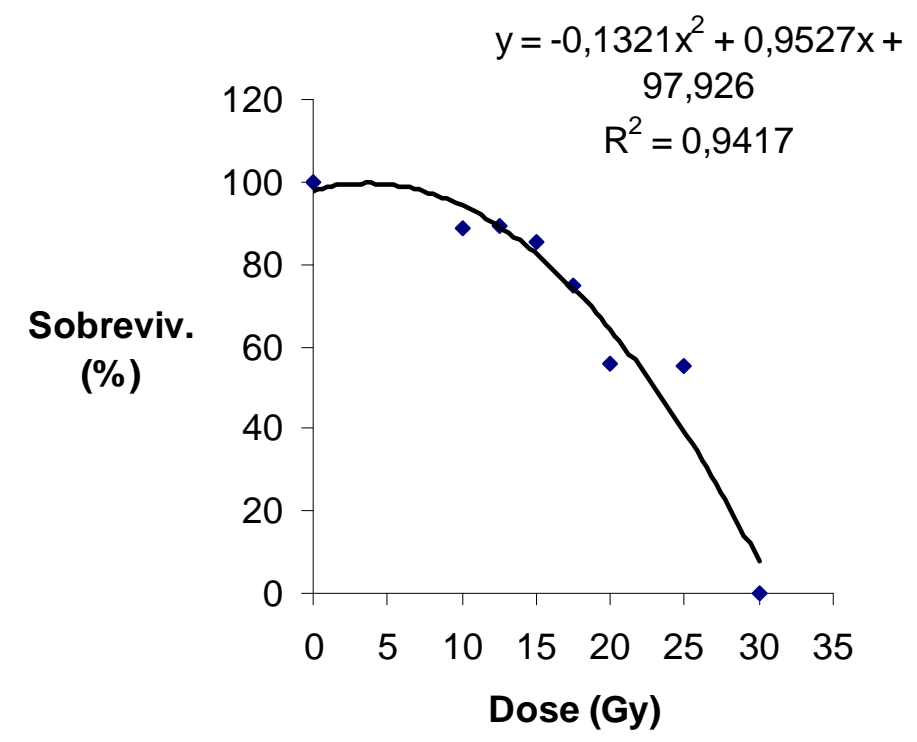

Figura 2. Sobrevivência das plantas da cultivar de crisântemo Cherry Dark, provenientes da irradiação de estacas nãoenraizadas, com várias doses de raios-gama. Avaliação realizada durante o florescimento (dados agrupados de sete experimentos, realizados de junho de 2001 a agosto de 2002). 
Tabela 1. Número e porcentagem de plantas sobreviventes, de mutantes e de plantas contendo mutação na coloração de inflorescência, após a irradiação com várias doses de raios-gama. Dados agrupados de sete experimentos

\begin{tabular}{|c|c|c|c|c|c|c|c|}
\hline \multirow[t]{2}{*}{$\begin{array}{l}\text { Dose } \\
\text { (Gy) }\end{array}$} & \multirow[t]{2}{*}{$\begin{array}{c}\text { Estacas } \\
\text { irradiadas }\end{array}$} & \multicolumn{2}{|c|}{$\begin{array}{c}\text { Plantas } \\
\text { Sobreviventes }\end{array}$} & \multicolumn{2}{|c|}{ Plantas mutantes (1) } & \multicolumn{2}{|c|}{$\begin{array}{l}\text { Plantas com mutações de } \\
\text { coloração de inflorescência }\end{array}$} \\
\hline & & $\mathrm{N}^{\circ}$ & $\%^{(2)}$ & $\mathrm{N}^{\mathrm{o}}$ & $\%^{(3)}$ & $\mathrm{N}^{\circ}$ & $\%^{(3)}$ \\
\hline 0,0 & 420 & 420 & 100,0 & 0 & 0,0 & 0 & 0,0 \\
\hline 10,0 & 760 & 676 & 88,9 & 531 & 78,6 & 405 & 59,9 \\
\hline 12,5 & 900 & 802 & 89,1 & 634 & 79,1 & 551 & 68,7 \\
\hline 15,0 & 620 & 529 & 85,3 & 410 & 77,5 & 316 & 59,7 \\
\hline 17,5 & 900 & 673 & 74,8 & 502 & 74,6 & 343 & 51,0 \\
\hline 20,0 & 760 & 424 & 55,8 & 282 & 66,5 & 115 & 27,1 \\
\hline 25,0 & 200 & 110 & 55,0 & 42 & 38,2 & 8 & 7,3 \\
\hline 30,0 & 200 & 0 & 0,0 & 0 & 0,0 & 0 & 0,0 \\
\hline
\end{tabular}

(1) Plantas mutantes - total de plantas com mutações na coloração e/ou de morfologia de inflorescência.

(2) Porcentagem em relação ao número de estacas irradiadas.

(3) Porcentagem em relação ao número de plantas sobreviventes, excluindo-se as do controle.

Tabela 2. Número total de plantas com mutação na coloração de inflorescência, número e porcentagem de plantas mutantes com quimeras mericlinal e periclinal, originadas de estacas irradiadas com várias doses de raios-gama. Dados agrupados de sete experimentos

\begin{tabular}{|c|c|c|c|c|c|}
\hline \multirow{2}{*}{$\begin{array}{l}\text { Dose } \\
\text { (Gy) }\end{array}$} & \multirow{2}{*}{$\begin{array}{l}\text { Plantas com mutação na } \\
\text { coloração de inflorescência }\end{array}$} & \multicolumn{2}{|c|}{ Mutante com quimera mericlinal $^{(1)}$} & \multicolumn{2}{|c|}{ Mutante com quimera periclinal ${ }^{(2)}$} \\
\hline & & $\mathrm{N}^{\mathrm{o}}$ & $\%(3)$ & $\mathrm{N}^{\circ}$ & $\%^{(3)}$ \\
\hline 0,0 & 0 & 0 & 0,0 & 0 & 0,0 \\
\hline 10,0 & 405 & 405 & 100,0 & 0 & 0,0 \\
\hline 12,5 & 551 & 551 & 100,0 & 0 & 0,0 \\
\hline 15,0 & 316 & 299 & 94,6 & 17 & 5,4 \\
\hline 17,5 & 343 & 322 & 93,9 & 21 & 6,1 \\
\hline 20,0 & 115 & 103 & 89,6 & 12 & 10,4 \\
\hline 25,0 & 8 & 6 & 75,0 & 2 & 25,0 \\
\hline 30,0 & 0 & 0 & 0,0 & 0 & 0,0 \\
\hline
\end{tabular}

(1) Mutante com quimera mericlinal - inflorescências com a cor original e uma ou mais colorações mutantes.

(2) Mutante com quimera periclinal - com todas as inflorescências contendo coloração única e diferente da original.

(3) Porcentagem em relação ao número de plantas com mutação na coloração de inflorescência. 
Tabela 3. Tipos diferentes de colorações de inflorescência em plantas mutantes, originadas de estacas irradiadas com várias doses de raios-gama. Dados agrupados de sete experimentos

\begin{tabular}{cc}
\hline Dose (Gy) & $\begin{array}{c}\text { Número de colorações diferentes observadas nas } \\
\text { inflorescências mutadas nos mutantes periclinais }{ }^{(1)}\end{array}$ \\
\hline 0 & 0 \\
10,0 & 0 \\
12,5 & 7 \\
15,0 & 9 \\
17,5 & 9 \\
20,0 & 2 \\
25,0 & 9 \\
\hline
\end{tabular}

(1) Mutante com quimera periclinal - com todas as inflorescências contendo coloração única e diferente da original. 